\title{
Minimally invasive subpial tonsillectomy for Chiari I decompression
}

\author{
Jeffrey S. Beecher ${ }^{1} \cdot$ Yong $\mathrm{Liu}^{2} \cdot$ Xiaoming $\mathrm{Qi}^{2} \cdot$ Paolo A. Bolognese ${ }^{3}$
}

Received: 3 March 2016 / Accepted: 14 June 2016/Published online: 5 July 2016

(C) The Author(s) 2016. This article is published with open access at Springerlink.com

\begin{abstract}
Background A number of different surgical techniques have been used through the years to address Chiari I malformation (CMI).

Methods This article describes how we surgically manage $\mathrm{CMI}$ at two high-volume centers. We call the technique the minimally invasive subpial tonsillectomy (MIST). The technique consists of a minimalistic dissection and craniectomy with a short, linear durotomy for the subpial tonsillar resection. The dura is closed without the use of a duraplasty. Conclusions We describe our current methods of surgery for CMI.
\end{abstract}

Electronic supplementary material The online version of this article (doi:10.1007/s00701-016-2877-2) contains supplementary material, which is available to authorized users.

Jeffrey S. Beecher

jeffrey.beecher@utsouthwestern.edu

Yong Liu

liuyong139@outlook.com

Xiaoming Qi

mingxqi@gmail.com

Paolo A. Bolognese

pbolognese@nspc.com

1 Department of Neurosurgery, UT Southwestern, 5323 Harry Hines Blvd, Dallas, TX 75390-8855, USA

2 Yuquan Hospital, Department of Syringomyelia, Tsinghua University, 5 Shijingshan Rd Shijingshan District, Beijing 100049 , China

3 Division of Neurosurgery, South Nassau Communities Hospital, 1 Healthy Way, Oceanside, NY 11572, USA
Keywords Chiari I malformation · Posterior fossa decompression $\cdot$ Cerebellar tonsil resection $\cdot$ Minimally invasive subpial resection $\cdot$ Syringomyelia

\section{Background}

Chiari I malformation (CMI) is defined by a small posterior fossa with herniation of the hindbrain through the foramen magnum [1-3]. A spectrum of surgical techniques has been utilized over the years to treat this disorder [2, 4-8]. All of them provide different degrees of decompression of the cervicomedullary junction. There remains controversy regarding which techniques are most effective in treating the pertinent anatomic pathology [2-6]. Some authors advocate for resection of the cerebellar tonsils, while others do not $[3,5,9]$.

Our technique for CMI is called minimally invasive subpial tonsillectomy (MIST). The technique consists of a minimalistic dissection, a small craniectomy, a $\mathrm{C} 1$ laminotomy, and a midline durotomy with subpial resection of the cerebellar tonsils. The dura is closed primarily. This technique originated at the Department of Syringomyelia in Beijing, China.

\section{Methods}

\section{Relevant surgical anatomy}

CMI surgery is centered on the junction of the foramen magnum and the posterior arch of $\mathrm{C} 1$. The cerebellar tonsils, by definition, will have herniated through the foramen magnum and often displaced well below C1. It is imperative that dissection appropriately uncovers the most inferior aspect of the tonsils. This is confirmed with intraoperative ultrasound that 
Fig. 1 a Intraoperative ultrasound demonstrating the cerebellar tonsil prior to durotomy and subpial resection. b Intraoperative ultrasound after MIST is performed to confirm adequate tonsillar resection

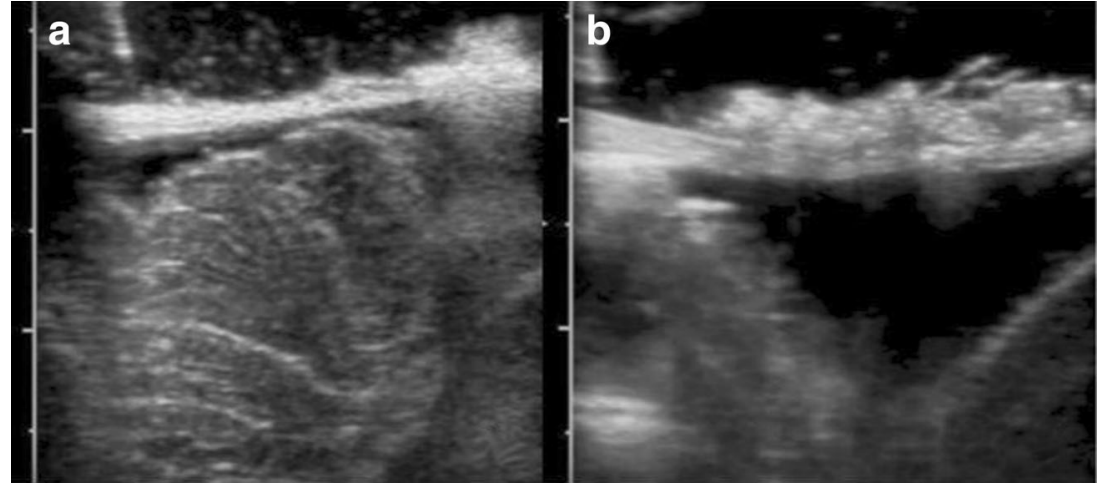

also reveals the neighboring anatomy of the cervicomedullary junction and the mass effect upon it from the tonsils. Intradurally, the tonsillar loops of the posterior inferior cerebellar arteries (PICAs) are in the midline, and running laterally are the spinal accessory nerves that also warrant decompression in many cases.

\section{Description of the technique}

The patient is positioned prone with the chin tucked with care not to kind the endotracheal tube, and the head is fixed with the Mayfield ${ }^{\circledR}$ skull clamp (Integra Life Sciences Corporation, Cincinnati, OH, USA). A $5-61 \frac{1}{2}-\mathrm{cm}$ midline skin incision is centered $1 \mathrm{~cm}$ above the craniocervical junction. Minimal electrocautery is used on the subcutaneous tissues, and is mainly reserved for the midline dissection of the linea alba. The narrow soft tissue dissection is followed with a small square-shaped suboccipital craniectomy $2 \mathrm{~cm}$ in length and 2-3 cm wide, followed by a $\mathrm{C} 1$ laminotomy (removal of the superior rim of the C1 lamina). A complete laminectomy is performed

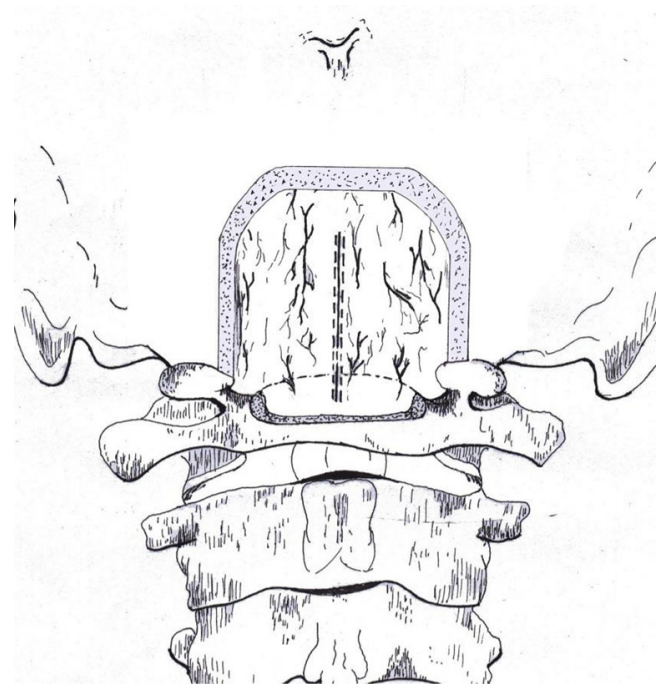

Fig. 2 Artistic rendering of the bone work and linear durotomy for MIST whenever the cerebellar tonsils are radiographically determined to be inferior to the arch of $\mathrm{C} 1$. Transdural intraoperative ultrasound is use to visualize the tonsils in the sagittal plane and this guides the durotomy length (Fig. 1a).

The linear, midline durotomy ranges between 2 and $3 \mathrm{~cm}$, and is performed under the microscope (Fig. 2). The dural edges are retracted with sutures. Surgical visualization is increased by movements of the operative table, and with gentle retraction of the dura with a nerve hook. The arachnoid is incised and resected, and CSF is drained. The PICA tonsillar loops are dissected from the inferior aspect of the tonsils.

Arachnoid bands are dissected off the tonsils to allow for mobility, and bipolar coagulation is then initiated along their posterior, medial, and inferior aspects. A horizontal incision is made along the mid-portion of the tonsils with sharp dissection. The tonsillar parenchyma is coagulated while gently suctioning away debris. The dissection is performed in a medial to lateral fashion. A number of vertically arranged septations are encountered within the tonsils, and these require sharp dissection. The pial surface of the tonsils will become increasingly mobile, and this allows for dissection of the

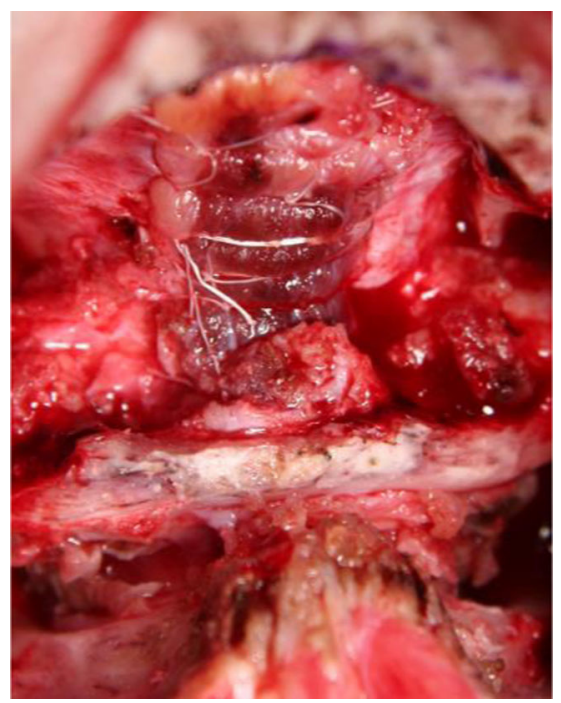

Fig. 3 Muscle patch sutured over the dural closure 
Fig. 4 a Preoperative and b postoperative MRI of a MIST procedure and it should be noted this patient concomitantly underwent a craniocervical fusion for basilar invagination

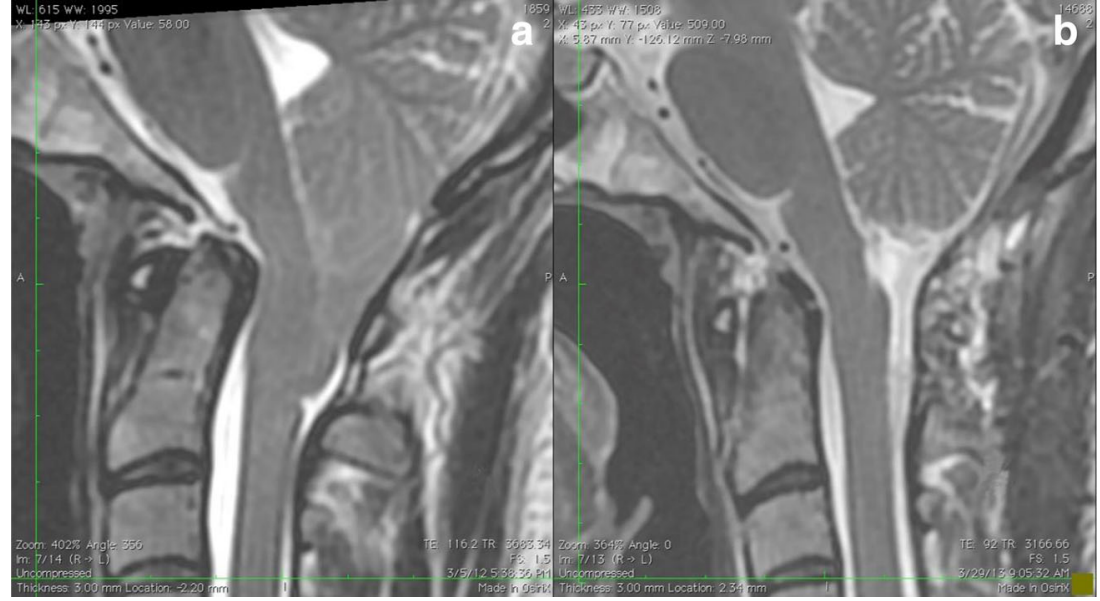

adhesions between the tonsils and the brainstem. The tonsils become progressively mobile and the lateral adhesions to the accessory nerve become accessible for dissection, while the tonsillar surface is more generously coagulated. The tonsillar resection is complete when: (1) Foramen of Magendie is unobstructed, (2) the cervicomedullary junction and the accessory nerves are decompressed, and (3) $3 / 4$ of the cerebellar tonsils are resected. This is confirmed with intraoperative ultrasound (Fig. 1b)

When possible, the pial edges surrounding the tonsillar incisions are re-approximated with a 7-0 Gore-Tex suture (Gore-Tex, W. L. Gore Inc., Flagstaff, AZ, USA). The dura is closed primarily with running unlocked 5-0 Gore-Tex sutures. A thin muscle patch is secured over the dural closure with 7-0 Gore-Tex interrupted sutures bridging over the muscle (Fig. 3). The anesthesia team provides Valsalva maneuvers to test the integrity of the closure (Video Supplement).

\section{Indications}

The indications for surgery are the presence of one or more of the following criteria:

1. Karnofsky score of 70 or less secondary to Chiari malformation stereotypic constellation of symptoms
2. An expanding syringomyelia on consecutive MRI scans, syringomyelia cavities in excess of $75 \%$ of the transverse cord diameter on the index MRI, or eccentric appearance of the syringomyelia cavity with intraparenchymal blebs

3. Severe, rapidly progressive neurological deficit

\section{Limitations}

There is an inherently longer learning curve in achieving the surgical goals through the described minimalistic approach. It is important to note that the resection of the cerebellar tonsils does come with risk. The direct surgical manipulation and resection of the tonsils can lead to a number of possible complications such as cerebellar edema or ischemia, injury to PICA, hemorrhage, brainstem or spinal cord injury, and cranial nerve injuries.

\section{How to avoid complications}

Above all, it is imperative that the dural edges are handled with care and the re-approximation is completed in a meticulous fashion. This, in combination with the muscle on-lay, dramatically reduces the risk of pseudomeningocele formation.
Table 1 Patient demographic information

\begin{tabular}{lll}
\hline General statistics & Beijing & NY \\
\hline Time range & $2004-2015$ & $2011-2015$ \\
Females/males & $969(64.8 \%) / 525(35.2 \%)$ & $151(83.8 \%) / 29(16.2 \%)$ \\
Mean age \pm SD & $39.3 \pm 10.8 \mathrm{SD}$ & $34.3 \pm 11.7 \mathrm{SD}$ \\
Age range & $2-76$ & $9-73$ \\
Pediatric/adults & $38(2.5 \%) / 1,456(97.5 \%)$ & $18(10.0 \%) / 162(90.0 \%)$ \\
Virgin/redo & $1490(99.7 \%) / 4(0.3 \%)$ & $139(77.2 \%) / 41(22.8 \%)$ \\
Total cases & $\mathbf{1 , 4 9 4}$ & $\mathbf{1 8 0}$ \\
\hline
\end{tabular}




\section{Specific perioperative considerations}

Preoperatively, it is imperative to study the patient's MRI and consider the length of the cerebellar tonsils herniation in relation to the posterior arch of $\mathrm{C} 1$ (Fig. 4a). This predicts how much of the $\mathrm{C} 1$ lamina will require removal to permit a useful durotomy.

Intraoperatively, aggressive subpial resection of the cerebellar tonsils provides direct decompression of the cervicomedullary junction as opposed to traditional techniques aimed at indirect decompression with excessive craniectomies or expansile duraplasties $[3-6,9]$. Furthermore, the minimal disruption of the soft tissues and bone may have an impact on wound healing and possibly decreasing the number of patients who progress to require a craniocervical fusion. The MIST technique has decreased our revision rate, which is likely secondary to the intraoperative evaluation of the tonsil resection. This provides immediate feedback regarding the cervicomedullary decompression that is confirmed with intraoperative ultrasound. Prior to closure of the dura, the anesthesia team is asked to administer one dose of $25 \mathrm{~g}$ of mannitol intravenously. Lastly, a primary closure is utilized and decreases the risk of pseudomeningocele formation.

Postoperatively, a 24-h course of dexamethasone $4 \mathrm{mg}$ intravenously every $6 \mathrm{~h}$ is prescribed. In combination with the intraoperative mannitol, this has been used to minimize any edema in the cerebellum from the surgery. The patient is monitored in the ICU for at least $24 \mathrm{~h}$, and early mobilization is initiated on postoperative day 1along with neck mobilization. An MRI is obtained to confirm the extent of resection and is compared to the preoperative study (Fig. 4b).

Perioperatively, we have found a very low complication rate (Tables 1 and 2), and believe MIST leads to the ideal patient outcomes.

\section{Specific information to give to the patient about surgery and potential risks}

Emphasis is placed on the inherent risk with resection of the cerebellar tonsils. This is in addition to the standard risk profile of the traditional operation.

Table 2 Complications

\begin{tabular}{lll}
\hline Complications & Beijing & NY \\
\hline Hydrocephalus & $7(0.4 \%)$ & $0(0.0 \%)$ \\
Meningitis & $3(0.2 \%)$ & $0(0.0 \%)$ \\
Permanent bulbar palsy & $4(0.3 \%)$ & $0(0.0 \%)$ \\
Cerebellar infarction & $3(0.2 \%)$ & $0(0.0 \%)$ \\
Cerebellar hemorrhage & $1(0.1 \%)$ & $0(0.0 \%)$ \\
Pseudomeningocele & $3(0.2 \%)$ & $0(0.0 \%)$ \\
Total & $\mathbf{2 1 ( 1 . 4 \% )}$ & $\mathbf{0}(\mathbf{0 . 0} \%)$ \\
\hline
\end{tabular}

\section{Key points}

- Preoperative MRI review for length of cerebellar tonsils

- Small craniectomy with minimal C1 lamina removal

- Midline, linear durotomy

- Maximize visualization of the cerebellar tonsils

- Subpial resection is performed from medial to lateral and is considered complete when:

- Foramen of Magendie is unobstructed

- No evidence of compression of the cervicomedullary junction

- $\quad \sim 3 / 4$ of the cerebellar tonsils have been resected

- Intraoperative ultrasound confirmation of surgical goals

- Primary closure of the dura with a muscle on-lay sutured in place

Acknowledgments The authors thank Dr. Thomas H. Milhorat for his valuable suggestions and encouragement.

Funding No funding was received for this research.

\section{Compliance with ethical standards}

Conflict of interest All authors certify that they have no affiliations with or involvement in any organization or entity with any financial interest (such as honoraria; educational grants; participation in speakers' bureaus; membership, employment, consultancies, stock ownership, or other equity interest; and expert testimony or patent-licensing arrangements), or non-financial interest (such as personal or professional relationships, affiliations, knowledge or beliefs) in the subject matter or materials discussed in this manuscript.

Ethical approval All procedures performed in studies involving human participants were in accordance with the ethical standards of the institutional and/or national research committee and with the 1964 Helsinki Declaration and its later amendments or comparable ethical standards.

Furthermore, this is a retrospective review and discussion of a technique so for this type of study formal consent is not required.

Open Access This article is distributed under the terms of the Creative Commons Attribution 4.0 International License (http:// creativecommons.org/licenses/by/4.0/), which permits unrestricted use, distribution, and reproduction in any medium, provided you give appropriate credit to the original author(s) and the source, provide a link to the Creative Commons license, and indicate if changes were made.

\section{References}

1. Attenello FJ, McGirt MJ, Gathinji M, Datoo G, Atiba A, Weingart J, Carson B, Jallo GI (2008) Outcome of Chiari-associated syringomyelia after hindbrain decompression in children: analysis of 49 consecutive cases. Neurosurgery 62:1307-1313, discussion 1313

2. Batzdorf U, McArthur DL, Bentson JR (2013) Surgical treatment of Chiari malformation with and without syringomyelia: experience with 177 adult patients. J Neurosurg 118:232-242 
3. Menezes AH (2011) Current opinions for treatment of symptomatic hindbrain herniation or Chiari type I malformation. World Neurosurg 75:226-228

4. Durham SR, Fjeld-Olenec K (2008) Comparison of posterior fossa decompression with and without duraplasty for the surgical treatment of Chiari malformation Type I in pediatric patients: a meta-analysis. J Neurosurg Pediatr 2:42-49

5. James HE, Brant A (2002) Treatment of the Chiari malformation with bone decompression without durotomy in children and young adults. Child's Nerv Syst : ChNS : Off J Int Soc Pediatr Neurosurg 18:202-206

6. Lazareff JA, Galarza M, Gravori T, Spinks TJ (2002) Tonsillectomy without craniectomy for the management of infantile Chiari I malformation. J Neurosurg 97:1018-1022
7. Munshi I, Frim D, Stine-Reyes R, Weir BK, Hekmatpanah J, Brown F (2000) Effects of posterior fossa decompression with and without duraplasty on Chiari malformation-associated hydromyelia. Neurosurgery 46:1384-1389, discussion 13891390

8. Mutchnick IS, Janjua RM, Moeller K, Moriarty TM (2010) Decompression of Chiari malformation with and without duraplasty: morbidity versus recurrence. J Neurosurg Pediatr 5:474-478

9. Milhorat TH, Bolognese PA (2003) Tailored operative technique for Chiari type I malformation using intraoperative color Doppler ultrasonography. Neurosurgery 53:899-905, discussion 905-896 